\title{
A Target HbA1c Between 7 and 7.7\% Reduces Microvascular and Macrovascular Events in T2D Regardless of Duration of Diabetes: a Meta-Analysis of Randomized Controlled Trials
}

Binayak Sinha $\cdot$ Samit Ghosal (D)

Received: March 16, 2021 / Accepted: April 13, 2021 / Published online: April 24, 2021

(C) The Author(s) 2021

\section{ABSTRACT}

Introduction: The target glycated haemoglobin (HbA1c) at which micro- and macrovascular benefits may be derived in type 2 diabetes (T2D) has never been clearly outlined. This metaanalysis was conducted on 15 randomized controlled trials to highlight the association of HbA1c range with outcomes.

Methods: The association of different HbA1c cluster (intention-to-treat (ITT) and end-ofstudy [EOS]) ranges $(\leq 6.5 \%, 6.6-7.0 \%$, $7.1-7.7 \%)$ with micro- and macrovascular complications and also the combined effect of T2D duration ( $<10$ years or $\geq 10$ years) and HbA1c levels was assessed.

Results: An intensive glucose-lowering strategy resulted in a significant $17 \% \quad(95 \% \quad \mathrm{CI}$ : $0.73-0.93, P<0.01)$ reduction in retinopathy, $18 \%$ reduction in macroalbuminuria $(95 \% \mathrm{CI}$ $0.62-0.83, P<0.01), 32 \%$ reduction in endstage renal disease (ESRD) (95\% CI 0.36-0.92, $P=0.02)$ and $13 \%$ reduction in non-fatal

Supplementary Information The online version contains supplementary material available at https:// doi.org/10.1007/s13300-021-01062-6.

B. Sinha

AMRI Hospitals, Kolkata, India

S. Ghosal ( $\square)$

Nightingale Hospital, Kolkata, India

e-mail: ramdasghosal@gmail.com myocardial infarction (NFMI) (95\% CI $0.78-0.96, P<0.01)$. Based on HbA1c achieved at EOS, a significant $46 \%$ reduction in retinopathy, $52 \%$ reduction in macroalbuminuria, 36\% reduction in (NFS) non-fatal stroke and a $22 \%$ reduction in all-cause mortality (ACM) were observed in the group with HbA1c in the $7.1-7.7 \%$ range. In the cohort, with diabetes duration $\geq 10$ years, reduction of HbA1c to $\leq 7.0 \%$ and significant improvements in new-onset retinopathy (24\%) and macroalbuminuria $(30 \%)$ were offset by an increase in ACM (21\%) and NFMI (17\%).

Conclusion: Contrasting with most recommendations, this meta-analysis including recent studies suggests that the optimal HbA1c range for $\mathrm{T} 2 \mathrm{D}$ is $7.1-7.7 \%$ regardless of diabetes duration.

Keywords: Meta-analysis; Type 2 diabetes; Glycated haemoglobin; Microvascular complications; Macrovascular complications; Diabetes duration 


\section{Key Summary Points}

Why carry out this study?

Control of HbA1C remains the main target to avoid complications of type 2 diabetes

There is no consensus as to what the target HbA1C in T2D should be with guidelines from august bodies based on divergent datasets differing extensively, confusing the practicing doctor

What was learned from this study?

The optimal range of target HbA1C in T2D regardless of duration should ideally be between 7 and $7.7 \%$

\section{DIGITAL FEATURES}

This article is published with digital features, including a summary slide to facilitate understanding of the article. To view digital features for this article go to https://doi.org/10.6084/ m9.figshare.14394608.

\section{INTRODUCTION}

According to the International Diabetes Federation Atlas of Diabetes, the number of people living with diabetes worldwide is expected to increase from approximately 415 million in 2015 to 642 million in 2040 [1]. The global increase in the prevalence of diabetes is associated with an increase in the incidence of diabetes-related complications. Macrovascular complications of diabetes are associated with higher mortality rates. The Global Burden of Disease report lists ischaemic heart disease and stroke as the top two causes of death amongst non-communicable diseases [2]. Indeed, ischaemic heart disease and cerebrovascular accidents together accounted for approximately $85.1 \%$ of cardiovascular (CV) deaths [2].
Given the magnitude of the disease burden, there is a need to determine optimal metabolic targets and establish concerted therapeutic strategies to achieve these targets. The UKPDS has clearly documented that a $0.9 \%$ reduction of $\mathrm{HbA1c}$ is associated with significantly reduced complications [3]. Though aware of the UKPDS findings, the American College of Physicians guidelines in 2018 were completely at odds with the other guidelines [4], leading to enormous confusion in physicians' minds as to what target they should be setting for each individual patient.

\section{Aim of This Meta-Analysis: To Determine the Optimal Target HbA1c Level to Prevent Type 2 Diabetes-Related Complications Based on the Available Evidence}

The above-mentioned guidelines recommended the target HbA1c levels based on the results of the UKPDS (33 and 34), ACCORD, ADVANCE and VADT trials [5-8]. However, there are other studies with similar aims that were not included in these analyses.

Moreover, data pertaining to the end-ofstudy (EOS) HbA1c level need to be included in the analysis, i.e., the HbA1c level achieved at the end of the study and not the level which was set as a target. For example, the STENO-2 trial aimed to achieve a HbA1c of $<6.5 \%$ (along with other stringent metabolic targets); however, only $15 \%$ of the patients achieved this target and the mean HbA1c at EOS was 7.7\% [9].

Our meta-analysis takes an in-depth look at all the available evidence and aims to decipher a reasonable and definitive HbA1c cut-off (if any) to prevent diabetes-related complications. We aimed to explore the following areas systematically:

- A relook at the outcome measures with any intensive glycaemic control strategy (irrespective of the HbA1c achieved at EOS).

- Analysis of the EOS outcomes. Different HbA1c ranges were analysed for outcomes.

- A subgroup analysis of the impact of diabetes duration and EOS HbA1c on outcomes. 


\section{METHODS}

This meta-analysis was conducted according to the recommendations of the PRISMA statement and was registered with PROSPERO (ID: CRD42 019122403) [10].

\section{Search Strategy}

A detailed literature search for relevant studies was conducted on the Cochrane Library, PubMed and Embase electronic biomedical databases. The following keywords were used: MeSH terms: 'type $2 \mathrm{DM}^{\prime}$; 'cardiovascular diseases'; 'diabetes retinopathy'; 'diabetes neuropathy'; 'diabetes nephropathy'; 'albuminuria'; 'renal insufficiency'. General terms: 'non-fatal myocardial infarction'; 'nonfatal stroke'; 'cardiovascular death'; 'all-cause mortality'; 'hospitalisation for heart failure'; 'hypoglycaemic agents'; 'glycaemic control'; 'tight glycaemic control'; 'intensive glycaemic control'. The citations retrieved were screened according to pre-specified criteria (Fig. 1). Prospectively designed studies with an intensive arm and a control arm were chosen for the final analysis $(n=15)$.

An important distinction made in this analysis was selection of both the micro- and macrovascular outcomes based on the current definitions (as used in recent CV outcomes trials). If the studies included had analysed the outcome of interest, but did not conform to the modern definition of the terms, they were not included in the meta-analysis.

\section{Data Extraction}

Both authors independently conducted a webbased search for citations dependent on the selected keywords. After identifying the citation from the web-based search, relevant data were extracted using the trial name, surname of the first author, year of publication, study population, place of origin of the study, design of the study, mean age, gender distribution, drugs in the intervention and control groups, dosages of agents in each group, background status related to cardiovascular disease and duration of follow-up. On identification of the basic database to work upon, further data extraction including the identification of retinal photocoagulation, vitrectomy, new-onset retinopathy, progressive retinopathy, diabetes-related blindness, new-onset or progressive diabetic peripheral neuropathy, new-onset or persistent macroalbuminuria, doubling of serum creatinine, ESRD, NFMI, NFS, CV death, ACM and hospitalisation for heart failure (hHF) was undertaken. Additional filters included were a cap on age $>18$ years and prospective clinical trials. No restrictions were placed based on language or date of publication. Any disagreements were resolved by conducting additional independent searches on a different day. After the initial process, a manual search was conducted jointly to identify the citations that met the inclusion criteria:

1. Randomised prospective trials on $\mathrm{T} 2 \mathrm{D}$

2. No cap on the number of patients recruited

3. Minimum duration of follow-up: 12 months

4. Reporting of the standardized end points in accordance with the accepted definitions as included in the cardiovascular outcomes trials (CVOTs).

5. The control group included standard of care or placebo. The other baseline metabolic as well as CV risk parameters should also be matched. The process of data extraction is detailed in Fig. 1.

\section{Quality Assessment}

The Cochrane risk of bias algorithm was used to assess quality of the studies (Supplementary material). The assessment of the individual component of the Cochrane risk of bias algorithm was based on the attributes of those parameters detailed by Higgins and Altman [11]. The authors (based on mutual consensus), after reviewing the materials and methods section of all the selected citations, agreed that eight studies scored an unclear risk of blinding of outcome assessment because of insufficient information on random sequence generation and allocation concealment. One study (Home 


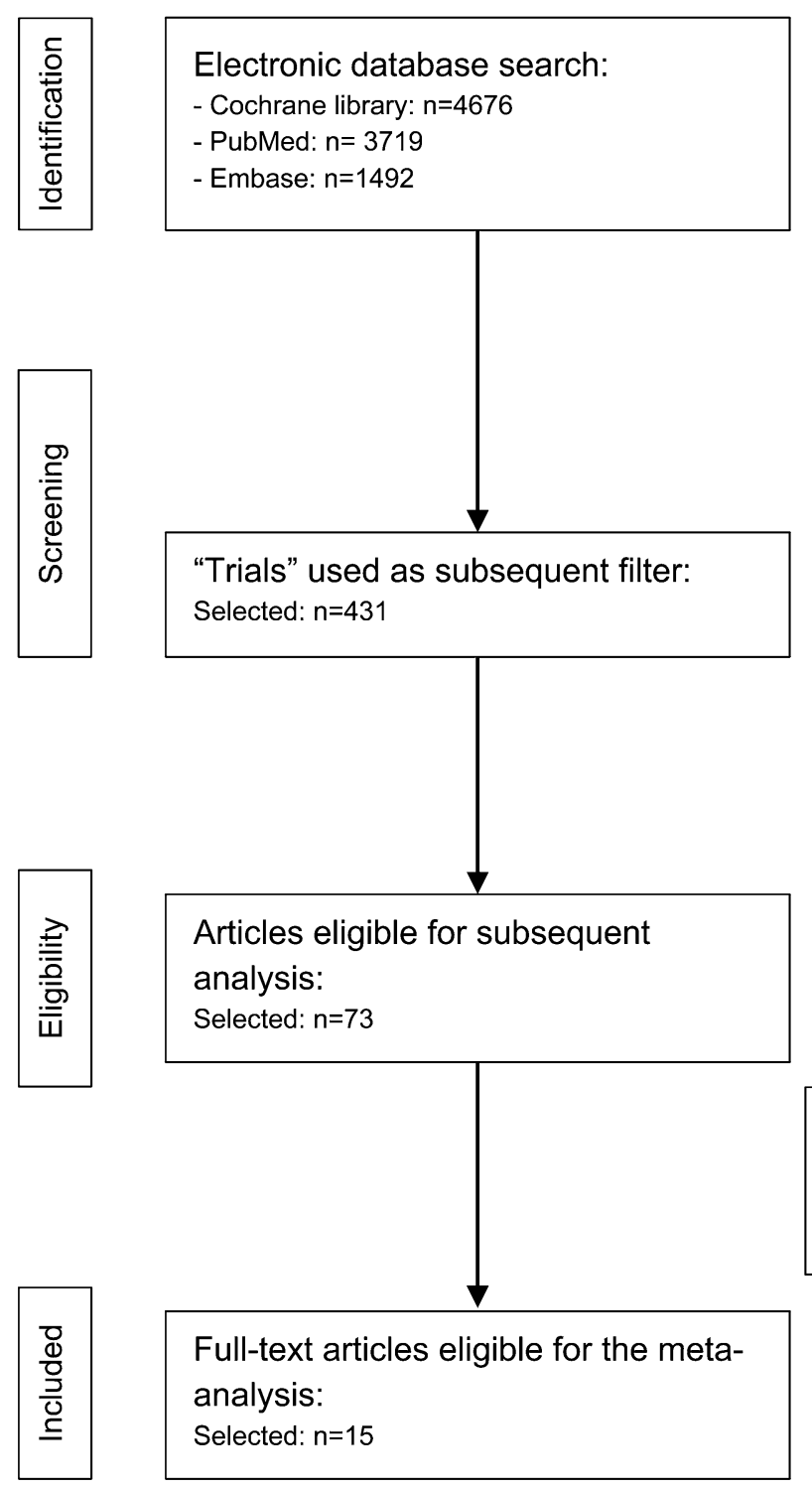

Fig. 1 Study selection process

et al.) showed bias due to blinding of outcome assessment, incomplete outcome data and other biases [12]. Issues related to unclear biases were also encountered in the UDGP, UKPDS 33 and 34, Veterans Affairs, ACCORD, ADVANCE and VADT trials [3, 5-8, 13, 14] (Supplementary material). An additional web-based search was conducted to locate the original published protocol of the citations included in the analysis. Comparing the intended outcomes to the ones finally reported helped in identifying selective reporting and other biases, namely
Articles excluded: ( $n=4245)$-Principle sourceCochrane library database:

- Non-randomized trials/commentaries/review articles

- Type 1 Diabetes Mellitus articles

- Articles on acute complications

Articles excluded: ( $n=358$ )-Principle sourceCochrane library database:

- Duplicate articles

- Non-relevent outcomes

Articles excluded: ( $n=58)$-Principle sourceCochrane library database:

- Based on inclusion criteria for meta-analysis biases related to non-declaration of funding and conflicts of interest, and the possibility of baseline imbalances (which is difficult to decipher because of the absence of publication of the trial protocol prior to conducting the trial, the degree of differences in the imbalances between the two comparative groups and a pre-adjudicated and pre-specified hierarchical testing protocol). In addition publication bias was assessed using funnel plots of the individual end points. 


\section{Data Synthesis and Analysis}

A detailed and up-to-date analysis of randomized prospective trials was conducted to assess the impact of intensive glycaemic control on retinal photocoagulation, vitrectomy, new-onset retinopathy, progressive retinopathy, diabetes-related blindness, new-onset or progressive diabetic peripheral neuropathy, new-onset or persistent macroalbuminuria, doubling of the serum creatinine level, ESRD, NFMI, NFS, CV death, ACM and hHF in comparison to conventional therapy. Since the aim was to compare the two different strategies, we did not restrict the inclusion criteria to control groups with placebo only. Having identified all the citations that reported the outcomes of interest, satisfying the pre-defined inclusion criteria, we proceeded with the meta-analysis.

Data analysis was conducted in a two-step manner:

Step 1: Analysis of the overall data (all 15 trials included) with an aim of identifying the impact of intensive glycaemic control versus conventional control on micro- and macrovascular outcomes based on the intension-to-treat strategy.

Step 2: A subgroup analysis was planned dividing the 15 included citations into two distinctive analytical strategy.

(a) The impact of intensive glycaemic control versus conventional control on micro- and macrovascular outcomes based on the endof-trial HbA1c value, i.e. based on the achieved HbA1c value. The rationale for using this strategy was based on the ACP guidance, which used the EOS HbA1c to propose a relaxed target for most $\mathrm{T} 2 \mathrm{D}$ patients.

(b) The impact of intensive glycaemic control versus conventional control on micro- and macrovascular outcomes based on the endof-trial HbA1c value and diabetes duration. Since a diabetes duration of $>10$ years is considered a high $\mathrm{CV}$ risk factor (Ismail Beigi et al. [15]), we took this cut-off as the parameter of interest.

A sensitivity analysis was planned for those parameters which demonstrated a significant impact associated with a high degree of heterogeneity defined as a $I^{2}>75$.

Data were analysed using the comprehensive meta-analysis software version 3 (Biostat Inc., Englewood, NJ, USA). Heterogeneity was assessed using the Cochrane Q and Higgins's $I^{2}$ test, and publication bias was assessed using funnel plots, with the precision (1/SE) plotted against the effect size. Individual effect size was assessed using a hazard ratio (HR) with $95 \%$ confidence interval (CI). Effect size was planned to be assessed using the fixed or the random model depending on heterogeneity or on the possibility of the analysed study containing the true effect. Significant heterogeneity was defined as a $P$ value $<0.1$ or a $I^{2}>75 \%$.

This article is based on previously conducted studies and does not contain any new studies with human participants or animals performed by any of the authors.

\section{RESULTS}

The meta-analysis was performed on a pooled population of 38,465 patients from 15 citations, with 20,247 individuals in the intensive therapy arm and 18,218 individuals in the conventional treatment arm. However, UKPDS 34 was a substudy of UKPDS 33, and different outcomes were reported in the Veterans Affairs and the VASCDM trials containing the same patient population. Hence, this meta-analysis was effectively performed on a pooled population of 37,559 individuals with 19,830 individuals in the intensive therapy arm and 17,728 individuals in the conventional treatment arm.

The baseline characteristics of the studies included are presented in Table 1 . The duration of the studies ranged from 2 to 10.7 years.

\section{Impact of (Positive/Negative/Neutral) Intensive Glucose-Lowering Strategy (Irrespective of EOS Achieved HbA1c) on Outcomes}

\section{Microvascular Outcomes}

An intensive glucose-lowering strategy resulted in a significant $17 \% \quad(95 \%$ CI $0.73-0.93$, $P<0.01)$ reduction in new-onset retinopathy or 


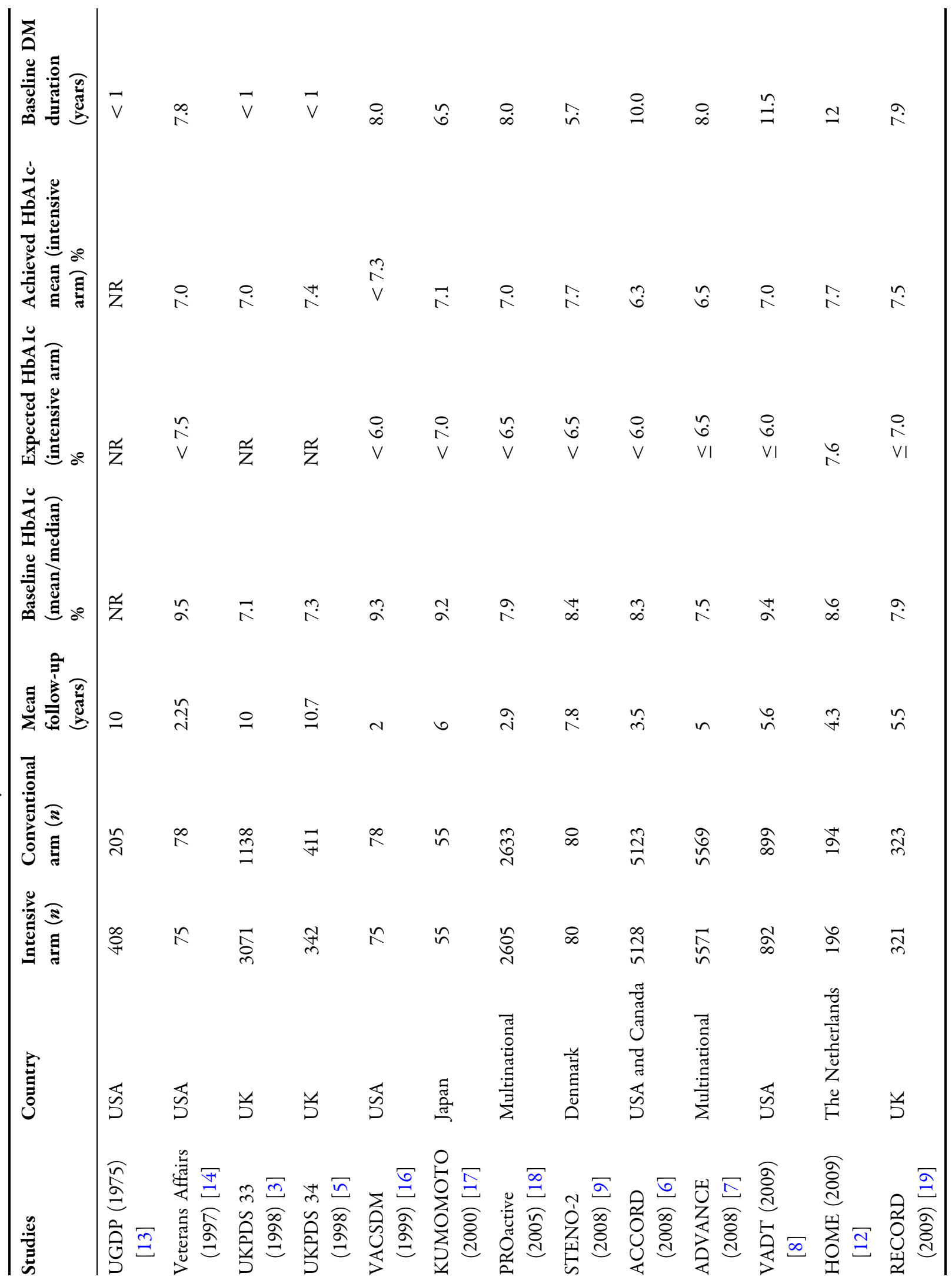




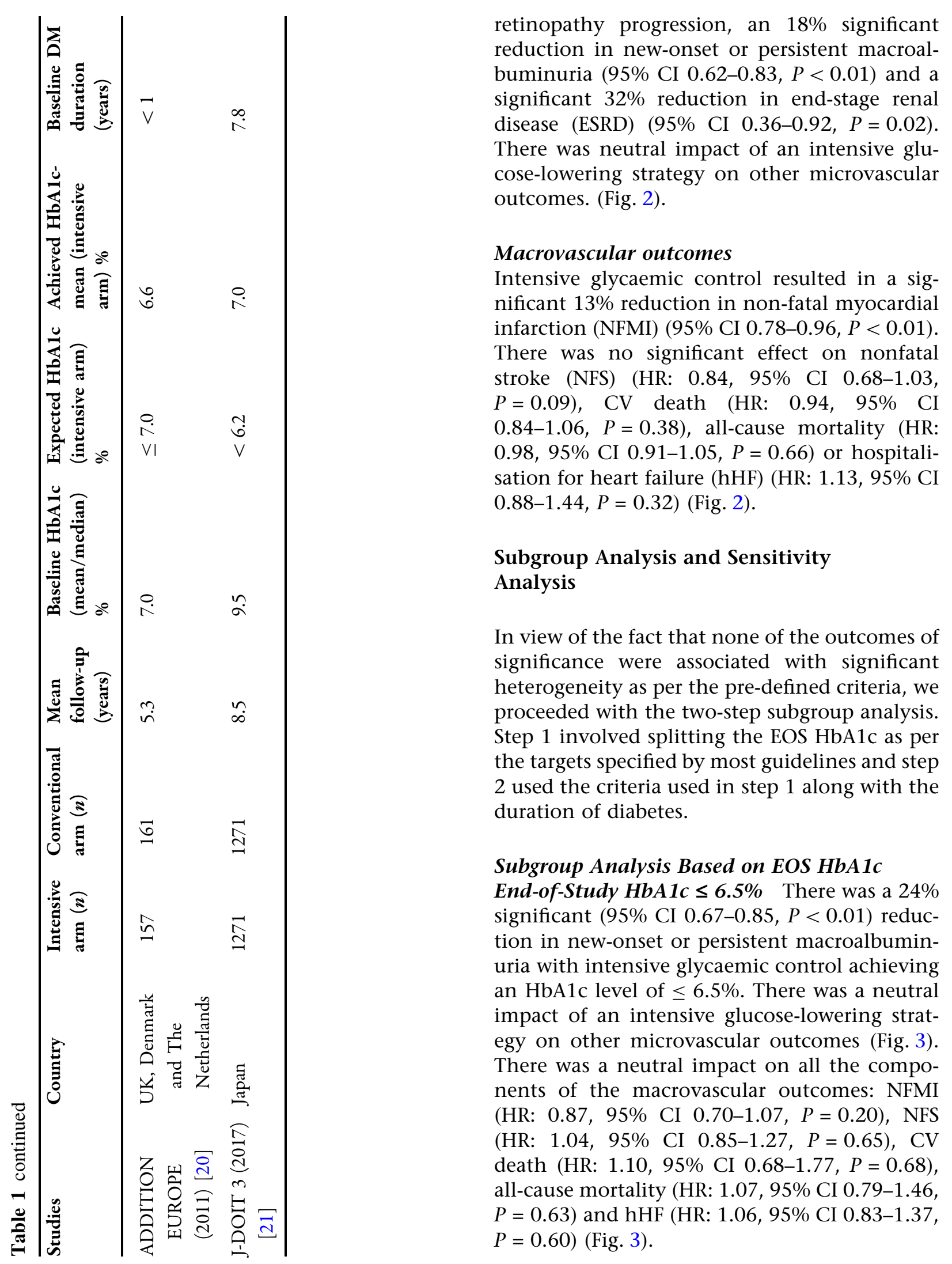


EOS HbA1c 6.6-7.0\% Intensive glycaemic control resulted in a significant $23 \%$ reduction in the requirement for retinal photocoagulation or vitrectomy (95\% CI 0.63-0.93, $P=0.008)$. There was a neutral impact of an intensive glucose-lowering strategy on other microvascular outcomes (Fig. 4).

There was a neutral effect of intensive glycaemic control on NFMI (HR: 0.85, 95\% CI 0.73-1.00, $P=0.05$ ), NFS (HR: 0.84, 95\% CI $0.69-1.10, P=0.07$ ), CV death (HR: $0.97,95 \%$
CI 0.76-1.25, $P=0.86$ ), all-cause mortality (HR: $1.00,95 \%$ CI $0.89-1.13, P=0.90)$ and hHF (HR: 1.15, 95\% CI 0.78-1.68, $P=0.46$ ) (Fig. 4).

EOS HbA1c 7.1-7.7\% There was a significant $46 \%$ reduction in new-onset or progressive retinopathy (95\% CI $0.35-0.79, P<0.01)$ and a significant $52 \%$ reduction in new-onset or persistent macroalbuminuria (95\% CI $0.30-0.76$, $P<0.01)$. There was a neutral impact of an
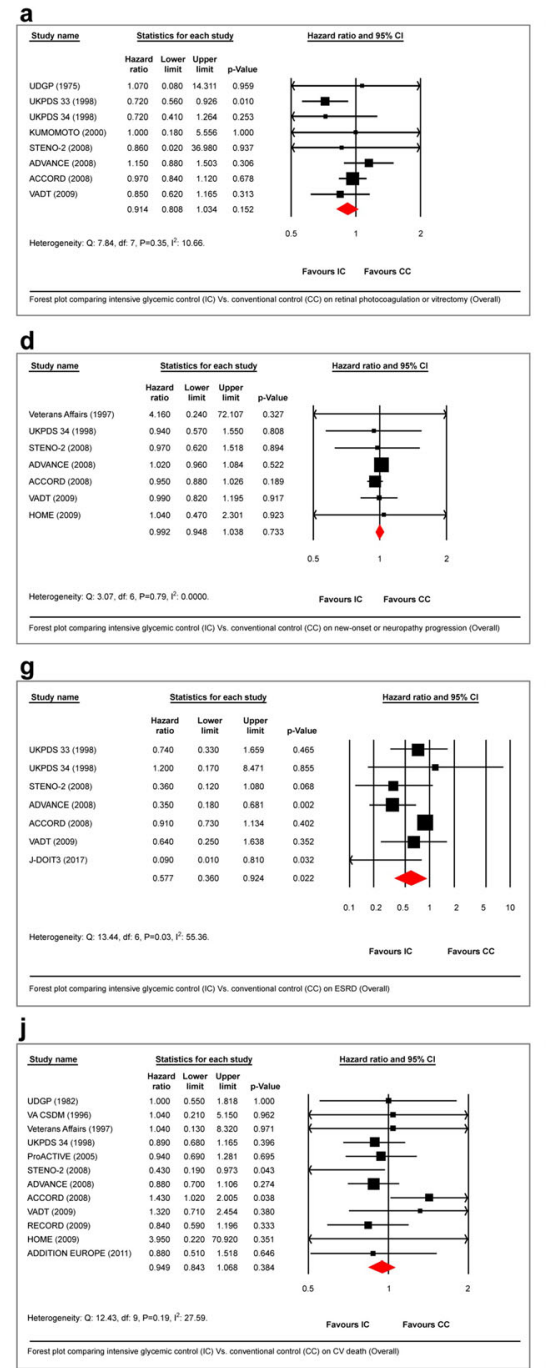

Fig. 2 Impact of intensive glycaemic control versus conventional glycaemic control on micro- and macrovascular complications (overall). a Retinal photocoagulation or vitrectomy. b Diabetes (DM)-related blindness. c Newonset or retinopathy progression. d New-onset or
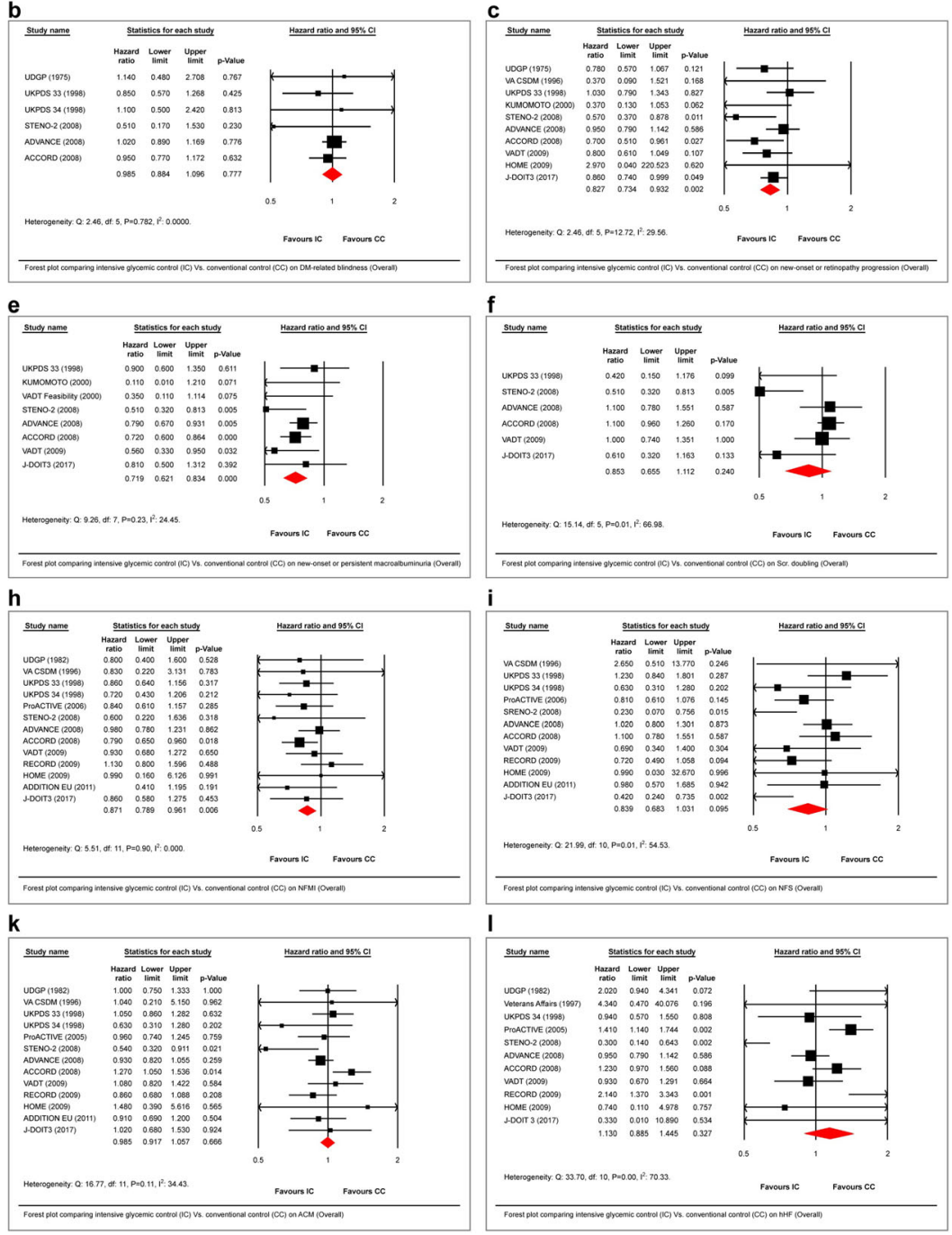

neuropathy progression. e New-onset or persistent macroalbuminuria. f Doubling of serum creatinine. g ESRD. h NFMI. i NFS. j CV death. $\mathbf{k}$ All-cause mortality. $1 \mathrm{hHF}$ 
intensive glucose-lowering strategy on other microvascular outcomes (Fig. 5).

There were no data pertaining to doubling of serum creatinine in this HbA1c range.

There was a significant $36 \%$ reduction in NFS (95\% CI 0.46-0.89, $P<0.01$ ) and a significant $22 \%$ reduction in all-cause mortality $(95 \% \mathrm{CI}$ $0.63-0.95, P=0.02$ ). The effects on NFMI (HR: $0.94,95 \%$ CI $0.71-1.24, P=0.69)$, CV death (HR: $0.83,95 \%$ CI $0.67-1.02, P=0.08$ ) and hHF (HR: $0.88,95 \%$ CI:0.32-2.41, $P=0.80$ ) were neutral (Fig. 5).

\section{Subgroup Analysis Based on EOS HbA1c and Diabetes Duration}

Intensive glycaemic control (HbA1c $\leq 7.0 \%)$ and $\mathrm{T} 2 \mathrm{D}$ duration $<10$ years resulted in a significant $19 \%$ reduction in new-onset or progressive macroalbuminuria and a significant 43\% reduction in ESRD. (Supplementary Material). The effects of intensive metabolic control on macrovascular complications were neutral. (Supplementary Material).

There was a significant $46 \%$ reduction in new-onset or progressive retinopathy and a significant $42 \%$ reduction in new-onset or persistent macroalbuminuria with intensive glucose lowering (HbA1c 7.1 to $7.7 \%$ ) and T2D duration $<10$ years. (Supplementary Material). Data pertaining to doubling of serum creatinine were available only for a single study (STENO2 ), which showed a significant $49 \%$ reduction. There was a significant $36 \%$ reduction in NFS achieved with intensive glycaemic control in patients with DM duration $<10$ years and HbA1c 7.1-7.7\%. (Supplementary Material).

In the subgroup represented by DM duration $\geq 10$ years and $\mathrm{HbA} 1 \mathrm{c} \leq 7.0 \%$, a $24 \%$ significant reduction in new-onset retinopathy, $30 \%$ significant reduction in new-onset macroalbuminuria and a significant 17\% reduction in NFMI were offset by a significant $40 \%$ increase in CV death and a $21 \%$ increase in ACM.

The combination of a T2D duration $\geq 10$ years and HbA1c $7.1-7.7 \%$ was represented by a single trial and hence could not be analysed.

\section{DISCUSSION}

Identification of the treatment target is the most important prerequisite to effective metabolic management. In the context of blood glucose management, the first evidence for such a target was provided by the UKPDS trial. In the UKPDS trial, a reduction of HbA1c by $0.9 \%$ from baseline translated into impressive microvascular benefits [3]. However, the microvascular endpoints which led to such impressive findings were driven by a reduction in rates of cataract extraction and albuminuria. The recent outcome studies, as well as recommendation committees, do not consider cataract extraction and microalbuminuria as standard components of microvascular outcomes [22].

The next spate of trials to define intensive glycaemic control was conducted a decade later. Of these, the results of the STENO-2 and ADVANCE trials attempted lowering the HbA1c levels to $<6.5 \%$, whereas ACCORD and VADT suggested a therapeutic target of $<6.0 \%$ [6-9]. Although, standardised outcome measures were analysed in these trials, there was considerable heterogeneity with respect to patient population, the duration of diabetes as well as the associated co-morbidities. This phenomenon likely explains the encouraging results obtained from the STENO-2 study, disappointing results from the ACCORD trial and neutral results from ADVANCE and VADT.

Despite differing baseline characteristics and inconsistent outcomes, different meta-analyses grouped all these studies together to determine the potential benefits of intensive glycaemic control over less stringent control.

However, the most important objection to grouping all these trials together is the divergent EOS HbA1c achieved in these trials. Most of the meta-analyses adopted the target HbA1c set at trial initiation as the definition of intensive control [23-25]. However, the EOS HbA1C values in all these studies were very different from the target HbA1C. For example, STENO-2 achieved a mean EOS HbA1c of $7.7 \%$ (target was $\leq 6.5 \%$ ), and VADT achieved a mean HbA1c of $7.0 \%$ at EOS (target was $\leq 6.0 \%$ ) 


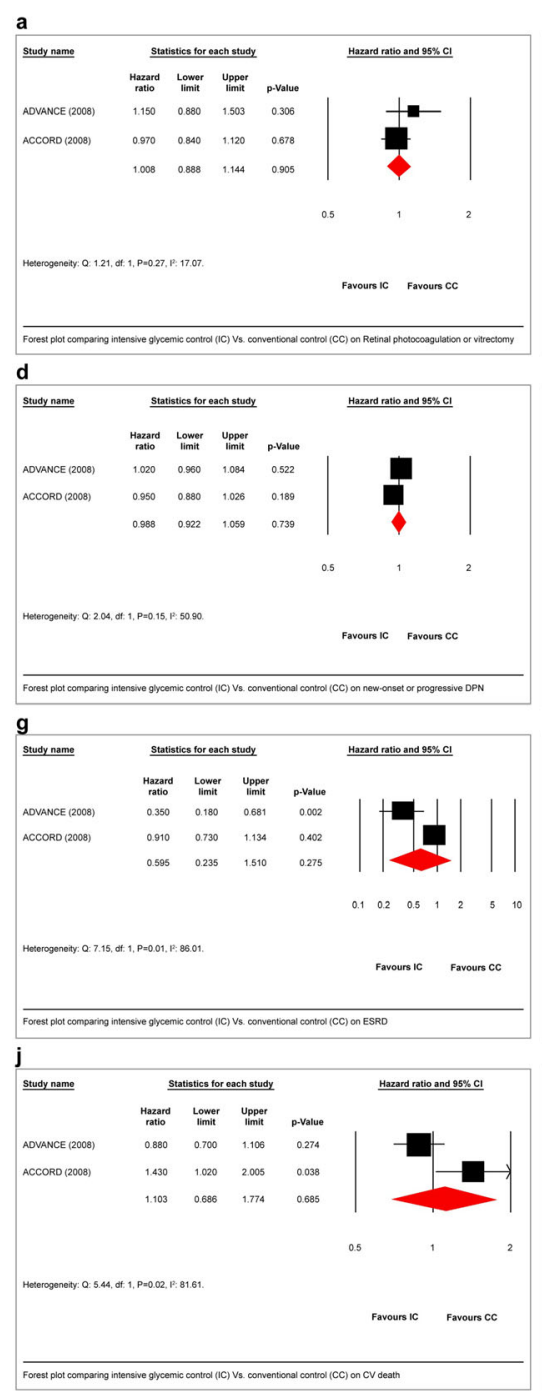

Fig. 3 Impact of intensive glycaemic control versus conventional glycaemic control on micro- and macrovascular complications (based on EOS HBAlc $\leq 6.5 \%$ ). a Retinal photocoagulation or vitrectomy. b Diabetes (DM)-related blindness. c New-onset or

Intuitively, this analytical strategy would seem inaccurate.

This problem is further compounded by the different HbA1c cut-off values proposed by the different diabetes guidelines. The 2018 ACP diabetes guidelines, which recommended a HbA1c range of $7.0-8.0 \%$ for most non-pregnant adults with T2DM, caused huge confusion amongst the physicians [4] because, in contrast, the 2021 ADA guidelines recommend a target

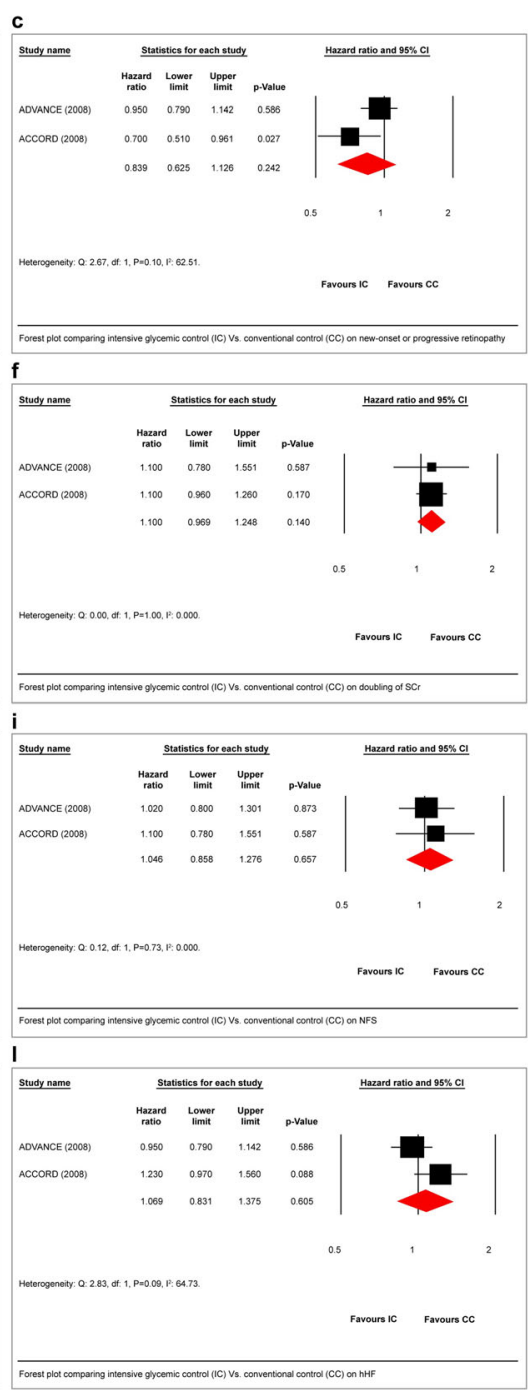

progressive retinopathy. $\mathbf{d}$ New-onset or progressive neuropathy. e New-onset or persistent macroalbuminuria. f Doubling of serum creatinine. g ESRD. h NFMI. i NFS. j CV death. $\mathbf{k}$ All-cause mortality. $1 \mathrm{hHF}$

HbA1c level of $<7.0 \%$, and the 2020 AACE guideline recommends $<6.5 \%$ for similar types of patients with diabetes $[26,27]$. The problem seems to stem from the fact that most of these analyses combined the results of only UKPDS, ACCORD, ADVANCE and VADT to arrive at a conclusion. However, there were many other trials during the same period and later which were not included in these analyses. 

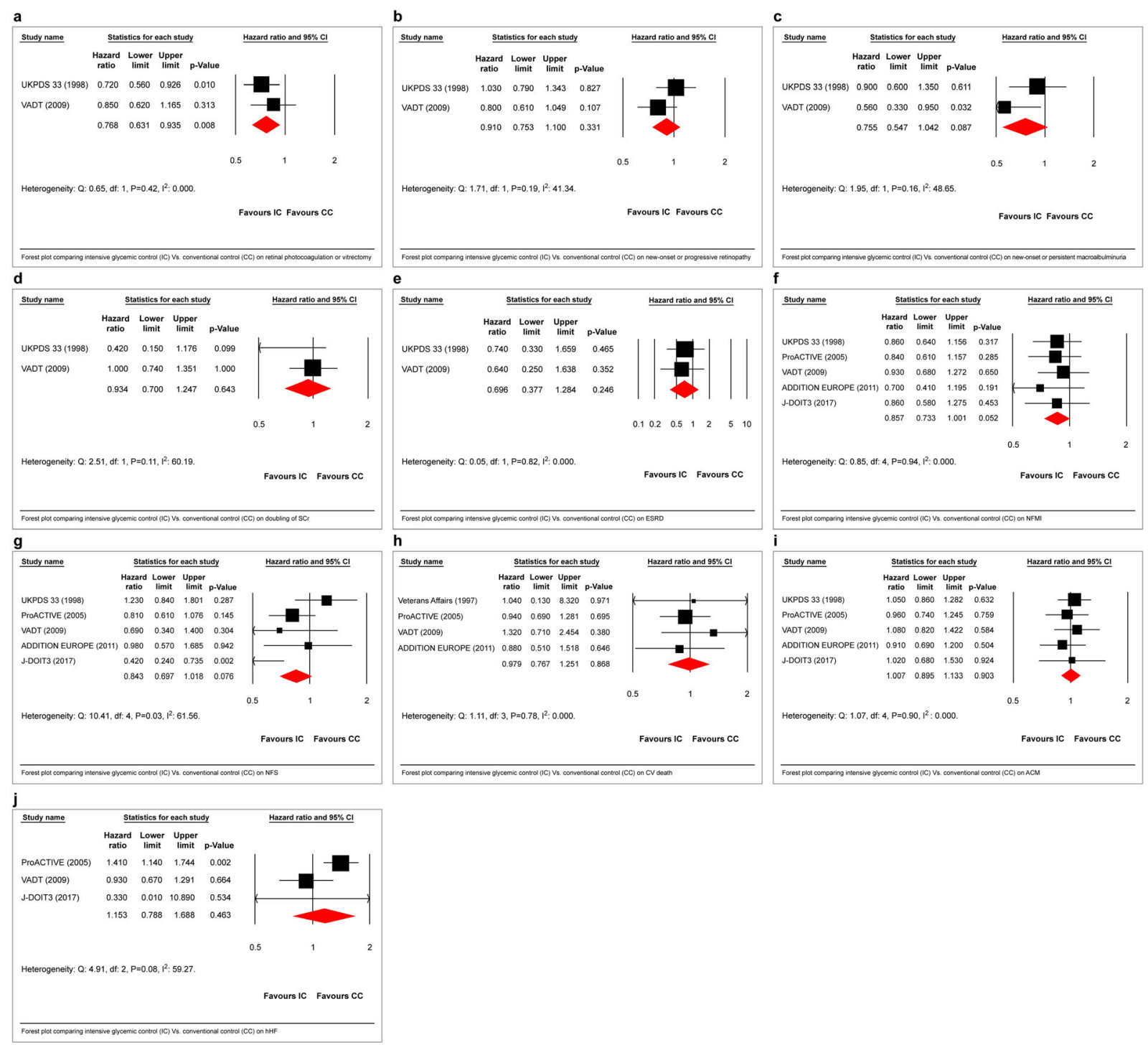

Fig. 4 Impact of intensive glycaemic control versus conventional glycaemic control on micro- and macrovascular complications (based on EOS HBAlc 6.6-7.0\%). a Retinal photocoagulation or vitrectomy. $\mathbf{b}$ New-onset or

Identification of the appropriate and accurate $\mathrm{HbA1c}$ level, a target which has a positive impact on micro- and macrovascular complications, necessitates assessment of different HbA1c ranges.

We conducted this meta-analysis in an attempt to overcome some of these shortcomings. As an initial strategy, we mimicked the pattern followed by previous meta-analyses and guidelines. The only difference was that we progressive retinopathy. c New-onset or persistent macroalbuminuria. d Doubling of serum creatinine. e ESRD. f NFMI. g NFS. h CV death. i All-cause mortality. $\mathbf{j}$ hHF

expanded the search to include all prospective trials to date and did not restrict our search up to 2008. Several smaller trials such as Kumamoto, Veterans Affairs and HOME were also included to increase the evidence base and to arrive at a more robust conclusion [12, 14, 17]. Even trials which primarily studied anti-diabetic agents, but divided the two comparative groups into intensive and conventional arms (PROactive and RECORD), were included to improve 


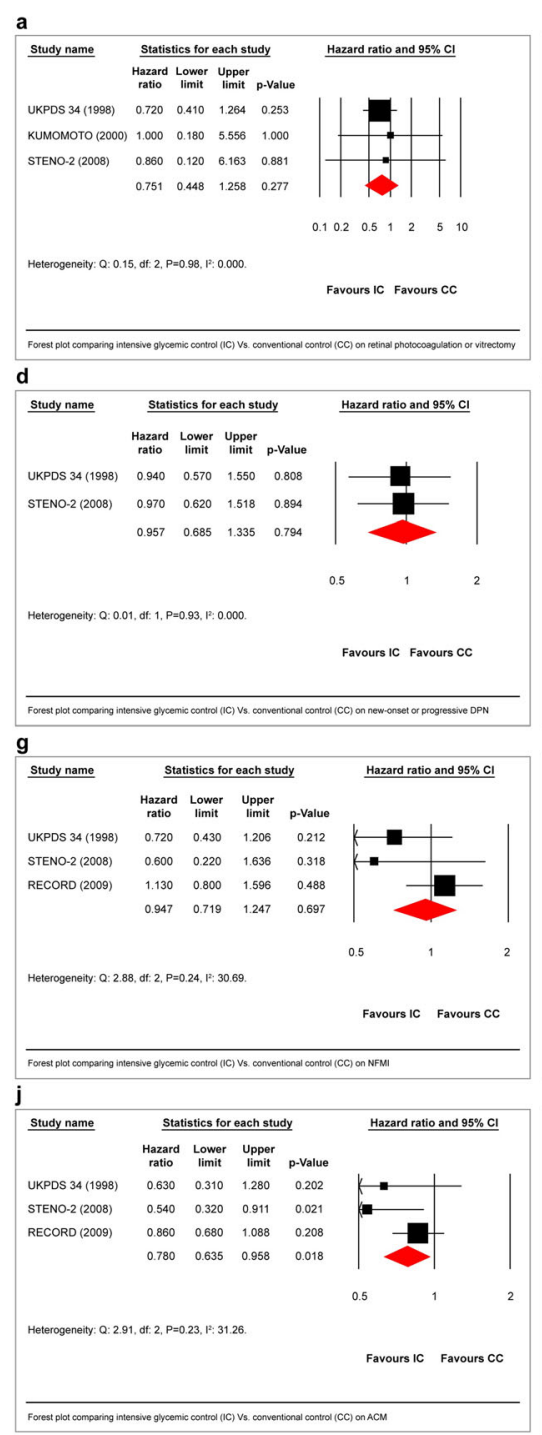

Fig. 5 Impact of intensive glycaemic control versus conventional glycaemic control on micro- and macrovascular complications (based on EOS HBA1c 7.1-7.7\%). a Retinal photocoagulation or vitrectomy. b DM-related

the yield [18, 19]. Thus, a total of 15 studies were included in the meta-analysis in contrast to the much smaller numbers in previous metaanalyses. The result suggested significant improvement in new-onset or progressive retinopathy, new-onset or persistent macroalbuminuria, ESRD and NFMI. This outcome with intensive glucose-lowering was encountered in a HbA1c target range of $<6.0-7.6 \%$, differing from an EOS HbA1C of 6.3-7.7\%. The question blindness. c New-onset or progressive retinopathy. $\mathbf{d}$ Newonset or progressive neuropathy. e New-onset or persistent macroalbuminuria. f ESRD. g NFMI. h NFS. i CV death. j All-cause mortality. $\mathbf{k}$ hHF

then is: how are the glycaemic targets set at $<$ $6.5 \%$ or even $7.0 \%$ in T2D?

To answer this question, we divided the HbA1c achieved at EOS into different ranges $(\leq 6.5 \%, 6.6-7.0 \%$ and $7.1-7.7 \%)$. Is the HbA1c $<6.5 \%$ justified as suggested by some diabetes guidelines? The only endpoint significantly impacted by targeting a HbA1c $<6.5 \%$ was new-onset or persistent macroalbuminuria ( $24 \%$ reduction). However, we need to 
appreciate the fact that only 2 out of the 15 studies achieved the targeted $\mathrm{HbA} 1 \mathrm{c} \leq 6.5 \%$ (ADVANCE and ACCORD). Targeting HbA1c between 6.6 and $7.0 \%$ resulted in a significant $23 \%$ reduction in new-onset or progressive retinopathy. However, the maximum benefit seems to be derived from a target $\mathrm{HbA1c}$ range of $7.1-7.7 \%$ (46\% reduction in new-onset or progressive retinopathy, $52 \%$ reduction in newonset or persistent macroalbuminuria, 36\% reduction in NFS and $22 \%$ reduction in ACM).

We have also included an EOS HbA1c of $7.1-7.7 \%$. Strange as it may sound, this is not without reason. This is because the HbA1C levels achieved at EOS in all the studies were consistently $\leq 7.7 \%$; hence, this was the basis of the upper limit of this range. A range of $7.1-7.5 \%$ and another range of $>7.5 \%$ could also have been chosen; however, in the absence of an adequate number of studies in these ranges, we decided to combine all the outcomes $\geq$ $7.1 \%$.

However, diabetes duration is another key determinant of the metabolic target. Long diabetes duration is arbitrarily considered to be $>10$ years owing to the significantly higher prevalence or risk of complications [15]. We included an additional strategy in our metaanalysis, i.e. a combination of $\mathrm{HbA} 1 \mathrm{c}$ range and the diabetes duration. Diabetes duration $<10$ years and achievement of HbA1c $\leq 7.0 \%$ were associated with a $24 \%$ lower risk of new-onset or persistent macroalbuminuria. Diabetes duration of $<10$ years and HbA1c level in the range $7.1-7.7 \%$ were associated with a more impressive $46 \%$ reduction in new-onset or progressive retinopathy, $42 \%$ reduction in newonset or persistent macroalbuminuria, $49 \%$ reduction in doubling of serum creatinine and $36 \%$ reduction in NFS. In contrast, those with aT2DM duration of $\geq 10$ years and who had achieved HbA1c $\leq 7.0 \%$ represented a mixed bag. There was a significant $24 \%$ reduction in new-onset or progressive retinopathy, $30 \%$ reduction in new-onset or persistent macroalbuminuria and $17 \%$ reduction in NFMI, but at the cost of a significant $40 \%$ increase in CV death and a $20 \%$ increase in ACM. Since only one study provided data pertaining to diabetes duration $\geq 10$ years and achievement of
HbA1c $\geq 7.1-7.7 \%$, no definitive conclusions could be drawn in this respect.

An observational study by Currie et al. aiming at treatment intensification with insulin documented a U-shaped curve for mortality [26]. In view of their findings the authors suggested a revision of guidelines to include a minimum HbA1c value. Our result and that of the observational study by Currie et al. seem to support the 2018 AFP guidelines as far as the desired HbA1c range for most non-pregnant adults with type 2 diabetes is concerned, in contrast to the ADA, AACE, IDF or NICE guidelines [4, 27-30].

\section{Study Limitations}

The different HbA1c ranges were not equally represented in the studies included in the analysis. However, this limitation is unavoidable since we had no other option but to work with the available and eligible trials. Hence, any sub-group analysis would be biased toward such an imbalance. Data pertaining to a few endpoints were reported by a single study; hence, it was difficult to arrive at a definitive conclusion. We would like to include those areas as part of our research recommendations. Inclusion of other risk factors apart from target HbA1c and duration of diabetes may have provided different results. This was not done in our metaanalysis. The main roadblock to such an approach was the limited number of prospective studies available for analysis. Any additional subgroups would have resulted in gross under-evaluation of the endpoints. The increase in $\mathrm{CV}$ death and ACM in the subgroup represented by DM duration $\geq 10$ years and HbA1c $\leq 7.0 \%$ could be due to the increased number of hypoglycaemic episodes in the intensive arm documented in the ACCORD trial. This could have skewed the data in the wrong direction. However, since the aim of this meta-analysis was to analyse the impact of an intensive versus convention therapeutic approach on outcomes, the mechanistic basis was not explored. Finally, the method of assessing HbA1c (JDS) was not the same as in the other included citations (NGSP method), which could have confounded 
some of the outcomes. However, after correcting for the difference as per the standardised equation $(\operatorname{NGSP}(\%)=1.02 \times$ JDS $(\%)+0.250)$, the KUMOTO study remained in the same target category (HbA1c range of $7.1 \%$ to $7.7 \%$ ). As a result, this did not alter the effect size analysis of the outcomes.

\section{Strengths of the Meta-Analyses}

One of the most prominent strengths of this meta-analysis was the inclusion of a large number of prospective studies. This allowed for analysis of one of the largest pools of data compared to most meta-analyses available to date. Another advantage was the inclusion of the HbA1c value achieved at EOS for analysis in contrast to the target $\mathrm{HbA1c}$ value used in some meta-analyses. We predominantly used the random effect model, which is one of the most conservative modes of analysis, for estimation of the effect size. This helped minimise the risk of over-estimation of the effect. Last but not the least, macrovascular endpoints were better represented in this meta-analysis, which is not surprising given the large number of trials that have focussed on CV safety and/or superiority.

\section{CONCLUSION}

In contrast to most diabetes guidelines that suggest an HbA1c target of $<6.5 \%$ or $<7.0 \%$ for most non-pregnant adults with T2DM, our meta-analysis suggests a target HbA1c in the range of $7.1-7.7 \%$ as the most reasonable, safe and evidence based. The results of this metaanalysis seem to endorse the 2018 ACP position with the exception of the HbA1c range of $7.0-8.0 \%$ since none of the trials achieved $\mathrm{HbA} 1 \mathrm{c}$ beyond $7.7 \%$ at EOS.

\section{ACKNOWLEDGEMENTS}

Funding. The journal's Rapid Service Fee was funded by the authors.
Authorship. All named authors meet the International Committee of Medical Journal Editors (ICMJE) criteria for authorship for this article, take responsibility for the integrity of the work as a whole, and have given their approval for this version to be published.

Authors' Contributions. Binayak Sinha conceptualised the idea and generated the hypothesis. Samit Ghosal, Binayak Sinha undertook the job of database search and study selection. Binayak Sinha, Samit Ghosal conducted the meta-analysis. Binayak Sinha edited the whole document prior to submission.

Disclosure. The authors (Binayak Sinha and Samit Ghosal) have nothing to disclose.

Compliance with Ethics Guidelines. This article is based on previously conducted studies and does not contain any new studies with human participants or animals performed by any of the authors.

Data Availability. Data sharing is not applicable to this article as no datasets were generated or analyzed during the current study.

Open Access. This article is licensed under a Creative Commons Attribution-NonCommercial 4.0 International License, which permits any non-commercial use, sharing, adaptation, distribution and reproduction in any medium or format, as long as you give appropriate credit to the original author(s) and the source, provide a link to the Creative Commons licence, and indicate if changes were made. The images or other third party material in this article are included in the article's Creative Commons licence, unless indicated otherwise in a credit line to the material. If material is not included in the article's Creative Commons licence and your intended use is not permitted by statutory regulation or exceeds the permitted use, you will need to obtain permission directly from the copyright holder. To view a copy of this licence, visit http://creativecommons.org/licenses/bync/4.0/. 


\section{REFERENCES}

1. International Diabetes Federation. Diabetes Atlas, 7th edn.https://www.idf.org/e-library/ epidemiology-research/diabetes-atlas.html. Accessed 25 Dec 2018.

2. GBD 2017 Causes of Death Collaborators. Global, regional, and national age-sex-specific mortality for 282 causes of death in 195 countries and territories, 1980-2017: a systematic analysis for the Global Burden of Disease Study 2017. Lancet. 2018;392: 1736-88. 6736(18)32203-7.

3. UK Prospective Diabetes Study(UKPDS) Group. Intensive blood-glucose control with sulphonylureas or insulin compared with conventional treatment and risk of complications in patients with Type 2 diabetes (UKPDS 33). Lancet. 1998;352: 837-53. https://doi.org/10.2337/db18-1629-P.

4. Qaseem A, Wilt TJ, Kansagara D, Horwitch C, Barry MJ, Forciea MA. Hemoglobin A1c targets for glycemic control with pharmacologic therapy for nonpregnant adults with Type 2 diabetes mellitus: a guidance statement update from the American college of physicians. Ann Intern Med. 2018;168: 569-76. https://doi.org/10.7326/M17-0939.

5. UK Prospective Diabetes Study (UKPDS) Group. Effect of intensive blood-glucose control with metformin on complications in overweight patients with Type 2 diabetes (UKPDS 34). Lancet. 1998;352: 854-65. https://doi.org/10.1016/S01406736(98)07037-8.

6. Action to Control Cardiovascular Risk in Diabetes Study Group, Gerstein HC, Miller ME, Byington RP, Goff DC Jr, Bigger JT, Buse JB, et al. Effects of intensive glucose lowering in Type 2 diabetes. N Engl J Med 2008;358:2545-59. https://doi.org/10. 1056/NEJMoa0802987.

7. ADVANCE Collaborative Group, Patel A, MacMahon S, Chalmers J, Neal B, Billot L, Woodward M, et al. Intensive blood glucose control and vascular outcomes in patients with Type 2 diabetes. N Engl J Med 2008;358:2560-72. https://doi.org/10.1056/ NEJMoa0802987.

8. Duckworth W, Abraira C, Moritz T, Reda D, Emanuele N, Reaven PD, et al. VADT Investigators. Glucose control and vascular complications in veterans with Type 2 diabetes. N Engl J Med. 2009; 360:129-39. NEJMoa0808431. https://doi.org/10.1056/

9. Gaede P, Lund-Anderson H, Parving H, Pederson O. Effect of multifactorial intervention on mortality in type 2 diabetes. N Engl J Med. 2008;358:580-91. https://doi.org/10.1056/NEJMoa0706245.

10. Details of the protocol for this systematic review were registered on PROSPERO and can be accessed at www.crd.york.ac.uk/PROSPERO/display_record. asp?ID=CRD42019122403.

11. Higgins JPT, Altman DG, Gøtzsche PC, Jüni $P$, Moher D, Oxman AD, et al. The Cochrane Collaboration's tool for assessing risk of bias in randomised trials. BMJ. 2011;343:d5928. https://doi. org/10.1136/bmj.d5928.

12. Kooy A, de Jager J, Lehert P, Bets D, Wulffelé MG, Donker AJ, et al. Long-term effects of metformin on metabolism and microvascular and macrovascular disease in patients with Type 2 diabetes mellitus. Arch Intern Med. 2009;169:616-25. https://doi.org/ 10.1001/archinternmed.2009.20.

13. University Group Diabetes Program. A study of the effects of hypoglycemic agents on vascular complications in patients with adult-onset diabetes: evaluation of phenformin therapy. Diabetes. 1975;24:65-184.

14. Abraira C, Colwell J, Nuttall F, Sawin CT, Henderson W, Comstock JP, et al. Cardiovascular events and correlates in the veterans affairs diabetes feasibility trial. Veterans affairs cooperative study on glycemic control and complications in Type II diabetes. Arch Intern Med. 1997;157:181-8. https:// doi.org/10.1001/archinte.1997.00440230053007.

15. Ismail-Beigi $\mathrm{F}$, Moghissi E, Tiktin M, Hirsch IB, Inzucchi SE, Genuth S. Individualizing glycemic targets in Type 2 diabetes mellitus: implications of recent clinical trials. Ann Intern Med. 2011;154: 554-9. https://doi.org/10.7326/0003-4819-154-8201104190-00007.

16. Azad N, Emanuele NV, Abraira C, Henderson WG, Colwell J, Levin SR, et al. The effects of intensive glycemic control on neuropathy in the VA cooperative study on Type II diabetes mellitus (VA CSDM). J Diabetes Complications. 1999;13:307-13. https:// doi.org/10.1016/S1056-8727(99)00062-8.

17. Shichiri M, Kishikawa H, Ohkubo Y, Wake N. Longterm results of the Kumamoto Study on optimal diabetes control in Type 2 diabetic patients. Diabetes Care. 2000;23:B21-9.

18. Dormandy JA, Charbonnel B, Eckland DJA, Erdmann E, Massi-Benedetti M, Moules I, et al. Secondary prevention of macrovascular events in patients with Type 2 diabetes in the PROactive study (Prospective pioglitAzone Clinical Trial in macroVascular Events): a randomized controlled trial. Lancet. 2005;366:1279-89. https://doi.org/10. 1016/S0140-6736(05)67528-9. 
19. Home PD, Pocock SJ, Beck-Nielsen H, Curtis PS, Gomis R, Hanefeld M, et al. Rosiglitazone evaluated for cardiovascular outcomes in oral agent combination therapy for Type 2 diabetes (RECORD): a multicentre, randomised, open-label trial. Lancet. 2009;373:2125-35. https://doi.org/10.1016/S01406736(11)60698-3.

20. Griffin S, Borch-Johnsen K, Davies MJ, Khunti K, Rutten GEHM, Sandbæk A, et al. Effect of early intensive multifactorial therapy on 5-year cardiovascular outcomes in individuals with Type 2 diabetes detected by screening (ADDITION-Europe): a cluster-randomised trial. Lancet. 2011;378:156-67. https://doi.org/10.1016/S0140-6736(11)60698-3.

21. Ueki K, Sasako T, Okazaki Y, Kato M, Okahata S, Katsuyama H, et al. Effect of an intensified multifactorial intervention on cardiovascular outcomes and mortality in Type 2 diabetes (J-DOIT3): an open-label, randomised controlled trial. Lancet Diabetes Endocrinol. 2017;5:951-64. https://doi. org/10.1016/S2213-8587(17)30327-3.

22. Food and Drug Administration: Guidance for Industry, Exposure-response relationships-study. Design, data analysis, and regulatory applications. https://www.fda.gov/downloads/Drugs/ GuidanceComplianceRegulatoryInformation/ Guidances/ucm072109.pdf. Accessed 28 Dec 2018.

23. Sardar P, Udell JA, Chatterjee S, Bansilal S, Mukherjee D, Farkouh ME. Effect of intensive versus standard blood glucose control in patients with Type 2 diabetes mellitus in different regions of the world: systematic review and meta-analysis of randomized controlled trials. J Am Heart Assoc. 2015;4:e001577. https://doi.org/10.1161/JAHA.114.001577.

24. Zhang X, Zhao J, Zhao T, Liu H. Effects of intensive glycemic control in ocular complications in patients with Type 2 diabetes: a meta-analysis of randomized clinical trials. Endocrine. 2015;49: 78-89. https://doi.org/10.1007/s12020-014-0459-8.

25. Boussageon $\mathrm{R}$, Bejan-Angoulvant $\mathrm{T}$, Saadatian-Elahi M, Lafont S, Bergeonneau C, Kassaï B, et al. Effect of intensive glucose lowering treatment on all-cause mortality, cardiovascular death, and microvascular events in Type 2 diabetes: meta-analysis of randomised controlled trials. BMJ. 2011;343:d4169. https://doi.org/10.1136/bmj.d4169.

26. Currie CJ, Peters JR, Tynan A, Evans M, Heine RJ, Bracco OL, et al. Survival as a function of $\mathrm{HbA}(1 \mathrm{c})$ in people with type 2 diabetes: a retrospective cohort study. Lancet. 2010;375(9713):481-9.

27. Targets G. Standards of medical care in diabetes2021. Diabetes Care. 2021;44(Supplement 1): S73-84. https://doi.org/10.2337/dc21-S006.

28. Garber AJ, Handelsman Y, Grunberger G, Einhorn D, Abrahamson MJ, Barzilay JI, et al. Consensus statement by the American Association of Clinical Endocrinologists and American College of Endocrinology on the comprehensive type 2 diabetes management algorithm-2020 executive summary. Endocr Pract. 2020;26(1):107-39. https:// doi.org/10.4158/CS-2019-0472.

29. International Diabetes Federation. Recommendations for managing type 2 diabetes in primary care. 2017.www.idf.org/managing-type2-diabetes.

30. NICE. 2017. Type 2 diabetes guideline, https:// www.nice.org.uk/guidance/ng28/chapter/1Recommendations. Accessed 28 Dec 2018. 